\title{
Impact of momentum anisotropy and turbulent chromo-fields on thermal particle production in quark-gluon-plasma medium
}

\author{
Vinod Chandra $^{1, \mathrm{a}}$, V. Sreekanth ${ }^{2, \mathrm{~b}}$ \\ ${ }^{1}$ Indian Institute of Technology Gandhinagar, Gandhinagar, Gujarat 382355, India \\ ${ }^{2}$ Centre for High Energy Physics, Indian Institute of Science, Bangalore 560012, India
}

Received: 22 December 2016 / Accepted: 15 June 2017 / Published online: 26 June 2017

(C) The Author(s) 2017. This article is an open access publication

\begin{abstract}
Momentum anisotropy present during the hydrodynamic evolution of the Quark-Gluon Plasma (QGP) in RHIC may lead to the chromo-Weibel instability and turbulent chromo-fields. The dynamics of the quark and gluon momentum distributions in this case is governed by an effective diffusive Vlasov equation (linearized). The solution of this linearized transport equation for the modified momentum distribution functions lead to the mathematical form of non-equilibrium momentum distribution functions of quarks/antiquarks and gluons. The modifications to these distributions encode the physics of turbulent color fields and momentum anisotropy. In the present manuscript, we employ these distribution functions to estimate the thermal dilepton production rate in the QGP medium. The production rate is seen to have appreciable sensitivity to the strength of the anisotropy.
\end{abstract}

\section{Introduction}

The experimental observation from the relativistic heavy-ion collisions at RHIC, BNL and LHC CERN has strongly suggested the creation of quark-gluon plasma (QGP) in a near perfect fluid state [1-7]. The space-time dynamics of the QGP has been modeled within the framework of second order relativistic dissipative hydrodynamics [8-16]. The hydrodynamical predictions for the collective flow coefficients and particle spectra in heavy-ion collisions (HIC) are seen to work well for hadronic probes. The role of hydrodynamics in HIC has been to convert the geometrical fluctuations in the initial geometry of the reaction zone (soon after the collisions) to a momentum anisotropy, which finally leads to collective flow in the hadronic observables. Therefore, the momentum anisotropy has been there during the entire space-time evo-

\footnotetext{
${ }^{a}$ e-mail: vchandra@iitgn.ac.in

b e-mail: sreekanth@chep.iisc.ac.in
}

lution of the QGP. On the other hand, the early stages of the HIC where the momentum distribution is far from equilibrium and highly anisotropic, leading to instabilities, has been well explored by several authors [17-19] along with a detailed study on its consequences. For a very recent review, we refer the reader to Ref. [20].

Based on the experimental observations, the QGP turned out to be a near perfect fluid with a tiny value for its shear viscosity to entropy ratio $(\eta / S \sim 1 / 4 \pi$, which is smallest among almost all the known fluids in nature). It has been realized that collisional processes among effective gluonic and quark degrees of freedom of the QGP alone could not explain such a small number. The presence of momentum anisotropy is seen to play dominant role in the substantial modulation of shear viscosity of the hot QCD medium in weak coupling regime [21-23] and may provide a possible explanation for the small, $\eta / S$. The main focus here is on the momentum anisotropy in the later stages of the collisions where the dynamics is determined by the hydrodynamics while the matter is near-equilibrated. The physics of such a momentum anisotropy is quite crucial for understanding the QGP medium, as in certain cases it may lead to the chromoWeibel instability in the QGP [24-26] and turbulent chromofields [21,22]. As mentioned in [27], the fields generated by such instabilities help in rapid isotropization of the parton distributions and drive the system to the hydrodynamical regime.

Instabilities have been extensively studied in the context of classical Yang-Mills dynamics and their role in thermalization and isotropization of the system [20] and their role in setting up the turbulence in the plasma. The two main frameworks for these investigations have been the classicalstatistical lattice [28-35] and CGC [36,37]. These classicalstatistical lattice simulations [28-31] revealed that, after an initial transient regime dominated by plasma instabilities and free streaming, the non-Abelian plasma exhibits the universal self-similar dynamics characteristic of wave turbulence. 
To handle the static case, Kurkela and Moore [38] proposed a new algorithm for solving the Yang-Mills equations in an expanding box that takes care of the transverse dynamics without getting affected by the longitudinal coarse lattices. The turbulence phenomenon has been studied in a static box by Kurkela and Moore [39] and Berges et al. [28-31] and they observed a cascade of energy flow towards higher momenta and the existence of a scaling solution therein. On the other hand, the CGC lattice simulations by Fukushima [36] and Fukushima and Gelis [37] have seen an energy flow from low to high wave number modes, eventually resulting in a spectrum consistent with Kolmogorov's power law indicating turbulent behavior. The later stages of the longitudinally expanding plasmas after instabilities have stopped growing were studied by Berges et al. [32-35] where they highlighted the role of quantum fluctuations in the classical lattice simulations and their role in deciding the time scales for the system to isotropize and approach thermal equilibrium. From all these above studies and a few more (for details we refer the reader to [20]), it is to be noted that the instabilities may lead to plasma turbulence. The role of instabilities and plasma turbulence might play a crucial role in understanding the properties of the QGP in heavy-ion collisions while one concentrates on the anisotropy (momentum) in the later stages of the collisions.

The physics of the chromo-Weibel instability (nonAbelian analog of the Weibel instability [40]) might play a crucial role in understanding the space-time evolution and properties of the QGP medium. The momentum anisotropy present during the hydrodynamic expansion of the QGP induces instability to the Yang-Mills field equations. The Weibel type of instabilities can be seen in the expanding QGP, since the width of the momentum component in the direction of the expansion gets squeezed by the expansion, leading to an anisotropic momentum distribution. The instability in the rapidly expanding QGP in heavy-ion collisions may also lead to the plasma turbulence [21,22]. Note that the plasma turbulence describes a random, non-thermal pattern of excitation of coherent color-field modes in the QGP. The power spectrum, in this case, turns out to be similar to that of vortices in a turbulent fluid [21,22].

An effective transport equation (Vlasov-Boltzmann) has been set up in $[21,22]$ in a form applicable to the case of the turbulent QGP by making additional assumptions regarding the field distributions in the Vlasov term. To reflect the turbulent nature a certain spatio-temporal correlation structure for fields at different space-time points (Gaussian form in $[21,22])$ has been considered. This allows one to rewrite the color-octet particle distribution function in the form of a dissipative term acting on the singlet distribution and eventually leads to a diffusive Vlasov-Boltzmann equation. Notably, the Vlasov (force term) operator thus obtained in the effective transport equation only picks up the contributions from the anisotropy. The color-electric field contributes through the thermal conductivity. The colormagnetic field mainly picks up the anisotropy in the plasma medium. Here, we are only dealing with the latter. It has already been realized that such turbulent color fields may contribute significantly to the transport processes in the QGP. As there is a significant decrease in the transport coefficients in the presence of turbulent fields [21,22,41], the small shear viscosity to the entropy ratio $(\eta / S)$ can perhaps be understood in terms of the dominance of such fields.

The primary goal here is to investigate the dilepton production rate in the presence of the chromo-Weibel instability. This could be done by first modeling the non-equilibrium momentum distribution functions $[42,43]$ that describe the expanding anisotropic QGP, followed by employing it to the kinetic theory description of dilepton production in the QGP medium. Our formalism is a straightforward extension of Refs. [21,22] for the interacting/realistic hot QCD equation of state (here the $(2+1)$-flavor lattice EoS is described in terms of a quasi-particle model). The hot QCD medium effects enter through the quasi-particle distribution functions along with the non-trivial energy dispersions. The near-equilibrium quasi-quark and quasi-gluon distributions that are employed here were obtained earlier in $[42,43]$ and utilized in the context of studying heavyquark dynamics in the anisotropic QGP/QCD medium. In this work, we employ them in exploring the dilepton production from the thermal QGP medium following the kinetic theory description of the dilepton production by $q \bar{q}$ annihilation at leading order within a $(1+1) \mathrm{d}$ boost invariant expansion of the thermal QGP medium in longitudinal direction.

This is perhaps the first time that the impact of the momentum anisotropy induced turbulent color fields has been included in the thermal particle production in hot QCD/QGP medium. As will be seen in the later part of the manuscript, such effects indeed play a significant role and cannot simply be ignored for the momenta and temperatures accessible in RHIC. It is to be mentioned that such anisotropy induced momentum distributions have already been exploited to investigate the heavy-quark dynamics in the QGP medium [42,43]. The heavy-quark dynamics gets a significant impact from such effects. Such effects may also be helpful to resolve the simultaneous estimation of $v_{2}$ and $R_{A A}$ for the heavy quarks [44].

Particles are produced from the thermal medium of an expanding fireball created in heavy-ion collisions, throughout its evolution, carrying crucial information as regards the state of the constituent matter [45-48]. The effects of the equations of state and non-equilibrium scenarios like dissipation etc. on such thermal particles from the QGP phase have been studied by various authors [49-55]. In this context 
it will be interesting to see how the presence of momentum anisotropy and turbulent chromo-fields will be affecting the thermal particle production.

The paper is organized as follows. In Sect. 2, the modeling of the non-equilibrium distribution function in the presence of an anisotropy driven instability is presented. Section 3 deals with the thermal particle productions rates in the anisotropic background medium and Sect. 4, discusses the yields in the presence of an expanding medium in heavy-ion collisions. In Sect. 5, conclusions and outlook are presented.

\section{Near (non)-equilibrium quark and gluon distributions}

Recall that the momentum anisotropy present in the quark and gluon momentum distribution functions induces instability in the Yang-Mills equations in a similar way to the Weibel instability in the case of electromagnetic plasmas. This instability, while coupled with the rapid expansion of the QGP, leads to anomalous transport and modulates the transport coefficients of the plasma substantially. This fact was realized by Dupree in the case of electromagnetic plasmas in 1954 [41] and later generalized for non-Abelian plasmas in Refs. [21,22,56]. In the context of QGP, the phenomenon of the anomalous transport is realized at the later stages of the collisions. Due to the hydrodynamic expansion of the QGP, one has appreciable momentum anisotropy present in the thermal distribution functions of quarks and gluons.

To obtain the near-equilibrium distribution within linearized transport theory we first need to have an adequate model for the isotropic (equilibrium) momentum distributions functions for the QGP degrees of freedom. To that end, we employ a quasi-particle description [57] of the latticebased QGP equation of state [58-62]. In this model, the forms of the equilibrium distribution functions, $f_{\text {eq }}$, are obtained by encoding the strong interaction effects in terms of effective fugacities for quarks/gluons $\left(z_{g, q}\right)$ :

$f_{0}^{g / q}=\frac{z_{g / q} \exp \left[-\beta E_{p}\right]}{\left(1 \mp z_{g / q} \exp \left[-\beta E_{p}\right]\right)}$,

where $p=|\vec{p}|, E_{p}=p$ for gluons and light quarks ( $u$ and $d)$. On the other hand for strange quark in $(2+1)$-flavor QCD, $E_{p}=\sqrt{p^{2}+m_{s}^{2}}$ for (s-quarks). Here, $m_{s}$ denotes the mass of the strange quark, and $\beta=T^{-1}$ denotes the inverse of the temperature. Since the model is valid for temperatures that are higher than $T_{c}$, we may ignore the strange quark mass effects. The sub/superscript q denotes the $u, d$ and $s$ quarks. The effective fugacities $\left(z_{g / q}\right)$ in the model are not merely temperature dependent parameters that encode the hot QCD medium effects; they lead to a non-trivial dispersion relation both in the gluonic and quark sectors as $\omega_{g, q}=E_{p}+T^{2} \partial_{T} \ln \left(z_{g, q}\right)$.

For a more detailed discussion of $z_{g, q}$, we refer the reader to Ref. [57]. This quasi-particle description of the hot QCD medium has shown to be highly useful in understanding the transport properties of the hot QCD medium [23,56,63,64], dilepton production in the QGP medium [55], and electrical conductivity and charge diffusion in the hot QCD medium [65].

It is worth to mention that there have been other quasiparticle descriptions in the literature, which could be characterized as effective mass models [66-69], effective mass models with gluon condensate [70-73], and effective models with a Polyakov loop [74-80]. The effective model with a Polyakov loop in [80] has been thermodynamically consistent. The model employed here is fundamentally distinct from all these models. In the presence of non-trivial quasiparticle dispersions (as in the case of the effective mass model or our model), the kinetic theory definition of the energymomentum tensor, $T^{\mu \nu}$, will get modified in terms of capturing the medium dependent terms [57,81-85]. Such modifications are mandated by the fact that $T^{\mu \nu}$ must incorporate the effects of the trace anomaly. This fact in the case of effective mass models has been described and an effective $T^{\mu \nu}$ is obtained in Ref. [83]. The authors furthermore estimated the viscosities of hot QCD matter [83]. There has been a more detailed study in this direction [87]. The mathematical expression for the modified $T^{\mu \nu}$ has been obtained for the current model in Ref. [63,64]. It is further to be noted that Bluhm et al. [86] highlighted the utility of the effective mass models for the relativistic heavy-ion collisions where non-zero baryon density aspects are also explored.

Next, we set up an effective transport equation for the nearequilibrium momentum distribution functions for quarks and gluons in the presence of an initial momentum anisotropy and space-time expansion of the QGP.

\subsection{An effective kinetic equation-the Dupree-Vlasov equation}

We start with the following ansatz for the non-equilibrium distribution function:

$f(\vec{p}, \vec{r})=\frac{z_{g, q} \exp \left(-\beta u^{\mu} p_{\mu}\right)}{1 \pm z_{g, q} \exp \left(-\beta u^{\mu} p_{\mu}+f_{1}(\vec{p}, \vec{r})\right)}$,

where $z_{g, q}$ are the effective gluon, quark fugacities coming from the isotropic modeling of the QGP in terms of lattice QCD equation of state and $u^{\mu}$ is the fluid four-velocity considering the fluid picture of the QGP medium. Here, $f_{1}(\vec{p}, \vec{r})$ denotes the effects from the anisotropy (momentum) to the equilibrium distribution function. To achieve the above mentioned near-equilibrium situation, $f_{1}$ must be a small perturbation. Under this condition, we obtain 


$$
f(\vec{p}, \vec{r})=f_{0}(p)+f_{0}\left(1 \pm f_{0}(p)\right) f_{1}(\vec{p}, \vec{r})+O\left(f_{1}(\vec{p}, \vec{r})^{2}\right) .
$$

The plus sign is for gluons and the minus sign is for the quarks/antiquarks.

Next, the following form for the ansatz is considered for the linear order perturbation to the isotropic gluon and quarks distribution functions, respectively:

$f_{1}(\vec{p}, \vec{r})=-\frac{1}{\omega_{g, q} T^{2}}\left(p_{i} p_{j} \Delta(\vec{p})(\nabla u)_{i j}\right)$,

where $f_{1}(\vec{p}, \vec{r}) \equiv f_{1}^{g, q}$ and $\Delta \equiv \Delta^{g, q}$. The quantity $\Delta$ captures the effects from the momentum anisotropy. In the local rest frame of the fluid (LRF) $f_{0}=f_{\mathrm{eq}}=\left(f_{0}^{g}, f_{0}^{q}\right)$, and considering longitudinal boost invariance [88], we obtain $\nabla \cdot \vec{u}=\frac{1}{\tau}$ and $\nabla u_{i j}=\frac{1}{3 \tau} \operatorname{diag}(-1,-1,2)$, leading to

$f_{1}^{g, q}=-\frac{\Delta^{g, q}(p)}{\omega_{g, q} T^{2} \tau}\left(p_{z}^{2}-\frac{p^{2}}{3}\right)$.

Let us now proceed to the set-up of the effective transport equation in the presence of turbulent chromo-fields that are induced by the momentum anisotropy in the thermal distribution of the quasi-gluons and quarks, while coupled with the rapid expansion of the QGP medium.

\subsubsection{Effective transport equation in turbulent chromo-fields}

The near-equilibrium (anisotropic) momentum distributions for the quasi-gluons and quasi-quarks in our case were obtained by solving the Vlasov-Boltzmann equation in the presence of turbulent chromo-fields in [23]. The approach has been based on a straightforward extension of the work Asakawa et al. [21,22]. Below, we offer the essential steps in the determination of the distributions.

The evolution of the quasi-quark and quasi-gluon momentum distribution functions in the anisotropic QGP medium with color fields can be obtained by setting up the VlasovBoltzmann equation [89]:

$v^{\mu} \frac{\partial}{\partial x^{\mu}} f(\mathbf{r}, \mathbf{p}, t)+g \mathbf{F}^{a} \cdot \nabla_{q} f^{a}(\mathbf{r}, \mathbf{p}, t)=0$

where $f(\mathbf{r}, \mathbf{q}, t)$ represents the parton distribution in phase space (sums over all parton colors), the quantities $\mathbf{p} \equiv \vec{p}$ and $\mathbf{r} \equiv \vec{r}$. Here, $f^{a}(\mathbf{r}, \mathbf{q}, t)$ denotes the color-octet distribution function. Note that here we are dealing with the collisionless plasma. Both distributions, $f$ and $f^{a}$, are defined in the semi-classical formalism in Refs. [90,91] as the moments of the distribution function $\tilde{f}(\mathbf{r}, \mathbf{p}, Q, t)$ in an extended phase space that includes the color sector:

$$
\begin{aligned}
f(\mathbf{r}, \mathbf{p}, t) & =\int \mathrm{d} Q \tilde{f}(\mathbf{r}, \mathbf{p}, Q, t), \\
f^{a}(\mathbf{r}, \mathbf{p}, t) & =\int \mathrm{d} Q Q^{a} \tilde{f}(\mathbf{r}, \mathbf{p}, Q, t) .
\end{aligned}
$$

Here $Q^{a}$ denotes the color charge, $v^{\mu}=\frac{p^{\mu}}{p^{0}}, p^{\mu}=\left(p^{0}=\right.$ $\left.E_{p}, \vec{p}\right)$. The color Lorentz force is defined as

$\mathbf{F}^{a}=E^{a}+\mathbf{v} \times B^{a}$.

The color-octet distribution function, $f^{a}$, will satisfy a transport equation which involves coupling with the phase space distributions of higher color-SU(3) representations. The near-equilibrium considerations allows us to truncate this hierarchy by keeping only the lowest order term in the gradients for both $f$ and $f^{a}$. The color-octet distribution identically vanishes at equilibrium. This implies that it is at least linear in perturbation. With these considerations, the transport equation for $f^{a}$ is obtained $[89,90]$ :

$v^{\mu} \frac{\partial f^{a}}{\partial x^{\mu}}+g f_{a b c} A_{\mu}^{b} v^{\mu} f^{c}+\frac{g C_{2}}{N_{c}^{2}-1} \mathbf{F}^{a} \cdot \nabla_{p} f=0$,

where $C_{2}$ and $f_{a b c}$ represent the quadratic Casimir invariant $\left(C_{2} \equiv\left(N_{c},\left(N_{c}^{2}-1\right) / 2 N_{c}\right)\right.$ and structure constants of $S U\left(N_{c}\right)$, respectively, and $A^{\mu}$ represents the gauge field.

Now the goal is to solve Eq. (11) and obtain $f^{a}$ in terms of $f$ and finally solve Eq. (7), in the case of turbulent chromofields. This has been done in [21,22] treating the isotropic hot QCD/QGP as an ultra-relativistic gas of the quarksantiquarks and gluons. In our case, the isotropic (local equilibrium) state is described in terms of effective quasi-gluon and quasi-quarks/antiquarks that describe the realistic hot QCD EoS (lattice) in the local rest frame of the QGP fluid. The extension of the whole treatment to the present case turned out to be quite straightforward. The interactions enter through the distribution functions and modified energy dispersions [23].

Following [21-23], we obtain an effective diffusive Vlasov-Boltzmann equation for the turbulent fields:

$v^{\mu} \frac{\partial}{\partial x^{\mu}} \bar{f}-\mathcal{F}_{V} \bar{f}=0$,

where

$$
\begin{aligned}
\mathcal{F}_{V}= & -\frac{g^{2} C_{2}}{4\left(N_{c}^{2}-1\right) \omega_{g, q}^{2}}\left\langle E^{2}+B^{2}\right\rangle \tau_{\mathrm{m}} \\
& \times\left[\left(\mathbf{L}^{(p)}\right)^{2}-\left(L_{z}^{(p)}\right)^{2}\right] .
\end{aligned}
$$

Here, $-i \mathbf{p} \times \nabla_{p}=\mathbf{L}^{(p)}, \mathbf{v}=\mathbf{p} / \omega_{g, q}$ and for $\bar{f}(\vec{p})$ we shall employ $f(\vec{p})$ in Eq. (1). Here, $\bar{f}$ denotes the ensemble averaged momentum distribution (singlet) function of quasipartons [21,22]. In our case, $\bar{f} \equiv f(\vec{p}, \vec{r})$, as given in Eq. 
(3). Note that we are only considering the anomalous transport and the collision term is not taken into account here. The argument here is based on the fact that the anomalous transport process, which leads to highly significant suppression of the transport coefficients in the expanding QGP, is the dominant mechanism as regards understanding the tiny value of the $\eta / S$ for the QGP. We intend to revisit it, employing an appropriate collision term in the near future. In obtaining the above mentioned diffusive Vlasov equation, the appropriate forms of the correlation functions (Gaussian correlators satisfy all the assumptions) for the color fields have been chosen [21,22], while assuming that the color-electric and -magnetic field are uncorrelated. Being real and symmetry properties of the Gaussian correlators with respect to two space-time points we express the chaotic nature of the hot QCD plasma. This is how the turbulent nature of the plasma and its effects on the dynamics through the transport equation is introduced here following Refs. [21,22].

The force term $\left(\mathcal{F}_{V}\right)$ in the case of a chromo-electromagnetic plasma will have the following form [21,22]:

$$
\begin{aligned}
\mathcal{F}_{V} \bar{f}(p) \equiv & \mathcal{F}_{V} f(\vec{p}, \vec{r}) \\
= & \frac{g^{2} C_{2}}{3\left(N_{c}^{2}-1\right) \omega_{g, q}^{2}}\left\langle E^{2}+B^{2}\right\rangle \tau_{m} \\
& \times\left[\left(\mathbf{L}^{(p)}\right)^{2}-\left(L_{z}^{(p)}\right)^{2}\right] f_{\text {eq }}\left(1 \pm f_{\text {eq }}\right) p_{i} p_{j}(\nabla u)_{i j}
\end{aligned}
$$

The quantities $\left\langle E^{2}\right\rangle$ and $\left\langle B^{2}\right\rangle$ are the color averaged chromoelectric and chromo-magnetic fields (average over the ensemble of turbulent color fields [21,22]), $\tau_{m}$ is the time scale for the instability.

Now, the action of the drift operator on $f_{\text {eq }}$ is given by

$$
\begin{aligned}
(v \cdot \partial) f_{\mathrm{eq}}= & -f_{\mathrm{eq}}\left(1+f_{\mathrm{eq}}\right)\left\{\left(p-\partial_{\beta} \ln \left(z_{g, q}\right)\right) v \cdot \partial(\beta)\right. \\
& +\beta(v \cdot \partial)(u \cdot p)\},
\end{aligned}
$$

where $p-\partial_{\beta} \ln \left(z_{g / q}\right) \equiv \omega_{g, q}$, is the modified dispersion relations. After some mathematical massaging, we obtain the following expression for the drift term:

$$
\begin{aligned}
(v \cdot \partial) & f_{\mathrm{eq}}(p) \\
= & f_{\mathrm{eq}}\left(1 \pm f_{\mathrm{eq}}\right)\left[\frac{p_{i} p_{j}}{\omega_{g, q} T}(\nabla u)_{i j}-\frac{m_{D}^{2}\left\langle E^{2}\right\rangle \tau^{\mathrm{el}} \omega_{g, q}}{3 T^{2} \partial \varepsilon / \partial T}\right. \\
& \left.+\left(\frac{p^{2}}{3 \omega_{g, q}^{2}}-c_{s}^{2}\right) \frac{\omega_{g, q}}{T}(\nabla \cdot \vec{u})\right],
\end{aligned}
$$

where $c_{s}^{2}$ is the speed of sound, $m_{D}^{2}$ is the Debye mass, $\varepsilon$ is the energy density, $\tau_{\mathrm{el}}$ is the time scale of the instability in chromo-electric fields. The expression is mathematically similar to $[21,22]$. The only difference is the appearance of a modified quasi-particle dispersion.
Finally, the effective Vlasov-Dupree equation (linearized) in the presence of turbulent color fields with the above ansatz is formulated in Refs. [21-23] and reads

$$
\begin{aligned}
& \left\{\left(\frac{p^{2}}{3 \omega_{g, q}}-c_{s}^{2}\right) \frac{\omega_{g, q}}{T}(\nabla \cdot \vec{u})+\frac{p_{i} p_{j}(\nabla u)_{i j}}{\omega_{g, q} T}\right\} f_{0}^{g, q}\left(1 \pm f_{0}^{g, q}\right) \\
& =\frac{g^{2} C_{2}}{3\left(N_{c}^{2}-1\right) \omega_{g, q}^{2}}\left\langle E^{2}+B^{2}\right\rangle \tau_{m} \mathcal{L}^{2} f_{1}^{g, q}(\vec{p}) f_{0}^{g, q}\left(1 \pm f_{0}^{g, q}\right) .
\end{aligned}
$$

Importantly, the first term in the left hand side of Eq. (17) contributes to the physics of isotropic expansion (bulk viscosity effects), which is not taken into account in the present work. As the analysis is valid for temperatures which are away from $T_{c}$, the bulk viscous effects can conveniently be neglected there.

Next, solving Eq. (17) for $\Delta$ analytically, we obtain the following expression $[63,64]$ :

$\Delta(\vec{p})=2\left(N_{c}^{2}-1\right) \frac{\omega_{g, q} T}{3 C_{2} g^{2}\left\langle E^{2}+B^{2}\right\rangle_{g, q} \tau_{m}}$.

The unknown factor, $\left\langle E^{2}+B^{2}\right\rangle_{g, q} \tau_{m}$, in the denominator of Eq. (18) can be related to the phenomenologically known quantity of the jet-quenching parameter, $\hat{q}$, in both gluonic and quark sectors as done in Ref. [92]. This connection is established as mentioned below. The two crucial transport coefficients that may get a significant contribution from the turbulent color fields are the shear viscosity, $\eta$, and the jet-quenching parameter, $\hat{q}$. Thus, the strength of the momentum anisotropy in the expanding QGP medium can be related to the physics of these parameters. Recall that the strength of the anisotropy, $\Delta(\vec{p})$, is related to $\eta$. The coefficient, $\eta$, is seen to be inversely proportional to $\hat{q}$ [21-23]. The jet-quenching parameter, $\hat{q}$, turns out to be proportional to the mean momentum square per unit length on the energetic parton imparted by turbulent fields [94]. In that context the anisotropy is related to the quenching. Here, we relate the unknown quantity $\left\langle E^{2}+B^{2}\right\rangle \tau_{m}$ - which encodes the physics of anisotropy and the chromo-Weibel instability - to $\hat{q}$ both in the gluonic and matter sectors as [92]

$\hat{q}=\frac{2 g^{2} C_{g / f}}{3\left(N_{c}^{2}-1\right)}\left\langle E^{2}+B^{2}\right\rangle \tau_{m}$,

where $C_{g}=N_{c}, C_{f}=\frac{\left(N_{c}^{2}-1\right)}{2 N_{c}}$ for the gluons and quarks, respectively. In the present case, we have $N_{c}=3$.

Employing the definition of $\hat{q}$ from Eq. (19) in Eq. (18), we obtain the following expression for the $\Delta$ term:

$\Delta=\frac{4 \omega_{g, q}^{2} T}{9 \hat{q}_{g, q}}$. 
With the above relation, we obtain the near-equilibrium distribution functions in terms of the jet-quenching parameter $\hat{q}$ as

$f^{g, q}(\vec{p})=f_{0}^{g, q}-f_{0}^{g, q}\left(1 \pm f_{0}^{g, q}\right) \frac{4 \omega_{g, q}}{9 \hat{q}_{g, q} T \tau}\left(p_{z}^{2}-\frac{p^{2}}{3}\right)$.

The jet-quenching parameter, $\hat{q}$, for both gluons and quarks has been estimated phenomenologically within several different approaches [93,95-99]. The gluon quenching parameter $\hat{q}_{g}$ can be obtained from the relation, $\hat{q}_{g} \equiv \frac{9}{4}$ (in terms of the Casimir invariants of the $S U$ (3) group). As we shall see later, such values of $\hat{q}_{g, q}$ induce very strong nonperturbative effects to the dileptons' spectra.

It is to be noted that we have only three independent functions, $z_{g}, z_{q}$, and $\hat{q}$ ( $\hat{q}$ for gluons and quarks are related by the respective Casimir invariants of $S U(3)$ ). The first two functions are estimated while describing the hot QCD equation of state. As stated earlier, the third one is a phenomenological parameter. Let us now proceed to the investigation of the thermal particle rates in the QGP medium.

It is important to note that momentum anisotropy and plasma instabilities in the initial stages of the RHIC have extensively been studied in the literature by several authors, while investigating the effects of color collective excitations [100-102] and thermalization [103] there. Rebhan et al. [104] studied the dynamics of non-abelian plasma instabilities within hard-loop dynamics and Romatschke and Venugopalan [105] explored the same within the CGC framework. The collective plasma modes in anisotropic QGP have been studied in [26,106-108]. The collisional energy loss in anisotropic QGP [109], momentum broadening [110], radiative energy loss [111], and the wake potential $[112,113]$ are some of the related effects that have been investigated in the anisotropic QGP. For recent reviews, we refer the reader to Refs. $[20,114]$ and the references therein.

\section{Modified thermal particle rates in QGP}

Particles are produced in all stages during fireball evolution in the heavy-ion collisions from locally thermalized QGP medium. The main focus here is to investigate dilepton pair in the thermal QGP medium via $q \bar{q}$-annihilation (most dominant source of dilepton pair production). As discussed in detail earlier, the momentum anisotropy present during the hydrodynamic evolution of the QGP medium in RHIC can be captured as the modification in the equilibrium (local) distributions of the gluonic and quark-antiquark degrees of freedom by setting up and solving an appropriate VlasovBoltzmann equation (linearized). The linear approximation is valid while the modifications are much smaller as compared to their equilibrium counterpart. In this near-equilibrium situation, we can still utilize the kinetic theory for obtaining particle production rates and yields. This could be done by just replacing the expressions for the momentum distributions as the modified ones as done below.

The major source of thermal dileptons in the QGP medium is the $q \bar{q}$ annihilation process, $q \bar{q} \rightarrow \gamma^{*} \rightarrow l^{+} l^{-}$. The rate of the dilepton production for this process can be written as [115]

$$
\begin{aligned}
\frac{\mathrm{d} R^{l^{+} l^{-}}}{\mathrm{d}^{4} p}= & \int\left[g f\left(\vec{p}_{1}\right) \frac{\mathrm{d}^{3} \vec{p}_{1}}{(2 \pi)^{3}}\right]\left[g f\left(\vec{p}_{2}\right) \frac{\mathrm{d}^{3} \vec{p}_{2}}{(2 \pi)^{3}}\right] \\
& \times \sigma\left(M^{2}\right) v_{r e l} \delta^{4}\left(p-p_{1}-p_{2}\right) .
\end{aligned}
$$

Here $p=\left(E=E_{1}+E_{2}, \vec{p}=\vec{p}_{1}+\vec{p}_{2}\right)$ is the fourmomentum of the dileptons and $p_{1(2)}=\left(E_{1(2)}, \vec{p}_{1(2)}\right)$ is that of quark or antiquark with $E_{1(2)} \simeq\left|\vec{p}_{1(2)}\right|$, while neglecting the quark masses. We denote the invariant mass of the virtual photon as $M^{2}=\left(E_{1}+E_{2}\right)^{2}-\left(\vec{p}_{1}+\vec{p}_{2}\right)^{2}$. The relative velocity of the quark-antiquark pair is given by $v_{\text {rel }}=\sqrt{\frac{M^{2}\left(M^{2}-4 m_{q}^{2}\right)}{4 E_{1}^{2} E_{2}^{2}}} \simeq \frac{M^{2}}{2 E_{1} E_{2}}$ and the term $\sigma\left(M^{2}\right)$ is the relevant thermal dilepton production cross section. With $N_{f}=2$ and $N_{c}=3$, we have $M^{2} g^{2} \sigma\left(M^{2}\right)=\frac{80 \pi}{9} \alpha^{2}$ [45]. The functions $f(\vec{p})$ are the quark and antiquark distribution functions with $g$ being the corresponding degeneracy factor. The expressions for the quark and antiquark distribution functions, obtained in the previous section, are

$f(\vec{p})=f_{0}-f_{0}\left(1-f_{0}\right) \frac{4 \omega_{q}}{9 \hat{q}_{q} T}\left(p_{i} p_{j} \Delta u_{i j}\right)$.

Since we are interested in the large invariant mass regime $M \gg T \gg m_{q}$, we can approximate the Fermi-Dirac distribution by a classical Maxwell-Boltzmann distribution, so that

$$
\begin{aligned}
f_{0}\left(\vec{p}_{i}\right) & \approx z_{q} \mathrm{e}^{-E_{i} / T} \\
f\left(\vec{p}_{i}\right) & \approx z_{q} \mathrm{e}^{-E_{i} / T}\left[1-\frac{4 \omega_{q}}{9 \hat{q}_{q} T}\left(p_{i} p_{j} \Delta u_{i j}\right)\right] .
\end{aligned}
$$

Keeping only up to the quadratic order in momenta, we can write the dilepton production rate as [53]

$$
\begin{aligned}
\frac{\mathrm{d} R}{\mathrm{~d}^{4} p}= & \int \frac{\mathrm{d}^{3} \vec{p}_{1} l^{-}}{(2 \pi)^{3}} \frac{\mathrm{d}^{3} \vec{p}_{2}}{(2 \pi)^{3}} \frac{M^{2} g^{2} \sigma\left(M^{2}\right)}{2 E_{1} E_{2}} \delta^{4}\left(p-p_{1}-p_{2}\right) \\
& \times f_{0}\left(\vec{p}_{1}\right) f_{0}\left(\vec{p}_{2}\right)\left[1-2 \frac{4 \omega_{q}}{9 \hat{q}_{q} T}\left(p_{1 i} p_{1 j} \Delta u_{i j}\right)\right] \\
= & \frac{\mathrm{d} R_{0}{ }^{l^{+} l^{-}}}{\mathrm{d}^{4} p}+\frac{\mathrm{d} R_{1}}{\mathrm{~d}^{4} p}
\end{aligned}
$$

The equilibrium contribution to the dilepton production

$$
\begin{aligned}
{\frac{\mathrm{d} R_{0} l^{+} l^{-}}{\mathrm{d}^{4} p}=} & \int \frac{\mathrm{d}^{3} \vec{p}_{1}}{(2 \pi)^{6}} \frac{M^{2} g^{2} \sigma\left(M^{2}\right)}{2 E_{1} E_{2}} \delta\left(E-E_{1}-E_{2}\right) \\
& \times z_{q}^{2} \mathrm{e}^{-\left(E_{1}+E_{2}\right) / T}
\end{aligned}
$$


is well known and is given by [55]

$$
{\frac{\mathrm{d} R_{0}}{\mathrm{~d}^{4} p}}^{l^{+} l^{-}}=\frac{z_{q}^{2}}{2} \frac{M^{2} g^{2} \sigma\left(M^{2}\right)}{(2 \pi)^{5}} \mathrm{e}^{-E / T} .
$$

Next, we calculate the non-equilibrium contribution to dilepton production, given as

$$
\begin{aligned}
{\frac{\mathrm{d} R_{1}}{\mathrm{~d}^{4} p}}^{l^{+} l^{-}}=- & -2 \int \frac{\mathrm{d}^{3} \vec{p}_{1}}{(2 \pi)^{6}} \frac{M^{2} g^{2} \sigma\left(M^{2}\right)}{2 E_{1} E_{2}} \delta\left(E-E_{1}-E_{2}\right) \\
& \times z_{q}^{2} \mathrm{e}^{-\left(E_{1}+E_{2}\right) / T}\left[\frac{4 \omega_{q}}{9 \hat{q}_{q} T}\left(p_{1 i} p_{1 j} \Delta u_{i j}\right)\right] \\
= & I_{i j}(p) \Delta u_{i j} .
\end{aligned}
$$

Here we have represented

$$
\begin{aligned}
I_{i j}(p)= & -2 \int \frac{\mathrm{d}^{3} \vec{p}_{1}}{(2 \pi)^{6}} \frac{M^{2} g^{2} \sigma\left(M^{2}\right)}{2 E_{1} E_{2}} \delta\left(E-E_{1}-E_{2}\right) \\
& \times z_{q}^{2} \mathrm{e}^{-\left(E_{1}+E_{2}\right) / T} \frac{4 \omega_{q}}{9 \hat{q}_{q} T} p_{1_{i}} p_{1 j} .
\end{aligned}
$$

Now, the most general form of this second rank tensor in LRF can be written as

$$
I_{i j}(p)=a_{0} \delta_{i j}+a_{2} p_{i} p_{j} .
$$

However, we note that on contraction with $\Delta u_{i j}$ only $a_{2}$ will survive as $\delta_{i j} \Delta u_{i j}=0$. By constructing the projection operator $Q_{i j}$ we can calculate $a_{2}=Q_{i j} I_{i j}$, where the form of the projection operator in LRF is given by

$Q_{i j}=-\frac{1}{2|\vec{p}|^{2}}\left(\delta_{i j}-\frac{3 p_{i} p_{j}}{|\vec{p}|^{2}}\right)$.

Now, the expression for the non-equilibrium contribution to the dilepton rate becomes

$$
\frac{\mathrm{d} R_{1}}{\mathrm{~d}^{4} p}{ }^{l^{+} l^{-}}=I_{i j}(p) \Delta u_{i j}=\left[Q_{m n} I_{m n}\right] p_{i} p_{j} \Delta u_{i j} .
$$

Computations of $Q_{m n} I_{m n}$ can be done using Eqs. (29) and (31),

$$
\begin{aligned}
Q_{m n} I_{m n}= & -2 \int \frac{\mathrm{d}^{3} \vec{p}_{1}}{(2 \pi)^{6}} \frac{M^{2} g^{2} \sigma\left(M^{2}\right)}{2 E_{1} E_{2}} \delta\left(E-E_{1}-E_{2}\right) \\
& \times z_{q}^{2} \mathrm{e}^{-\left(E_{1}+E_{2}\right) / T} \frac{4 \omega_{q}}{9 \hat{q}_{q} T} p_{1 m} p_{1 n} \\
& \times\left[-\frac{1}{2|\vec{p}|^{2}}\left(\delta_{m n}-\frac{3 p_{m} p_{n}}{|\vec{p}|^{2}}\right)\right] \\
= & -\frac{8}{9} \frac{1}{\hat{q}_{q} T} \int \frac{\mathrm{d}^{3} \vec{p}_{1}}{(2 \pi)^{6}} \frac{M^{2} g^{2} \sigma\left(M^{2}\right)}{2 E_{1} E_{2}} \delta\left(E-E_{1}-E_{2}\right) \\
& \times z_{q}^{2} \mathrm{e}^{-\left(E_{1}+E_{2}\right) / T}\left\{p_{1}+T^{2} \partial_{T} \ln \left(z_{q}\right)\right\} \\
& \times\left[-\frac{\left|\vec{p}_{1}\right|^{2}}{2|\vec{p}|^{2}}+\frac{3\left(\vec{p} \cdot \vec{p}_{1}\right)}{2|\vec{p}|^{4}}\right] \\
= & -\frac{8}{9} \frac{1}{\hat{q}_{q} T}[\mathscr{M}+\mathscr{N}],
\end{aligned}
$$

where we have used Eq. (2): $\omega_{q}=p+T^{2} \partial_{T} \ln \left(z_{q}\right)$. Next, we calculate the integrals $\mathscr{M}$ and $\mathscr{N}$ to find

$$
\begin{aligned}
\mathscr{M}= & \int \frac{\mathrm{d}^{3} \vec{p}_{1}}{(2 \pi)^{6}} \frac{M^{2} g^{2} \sigma\left(M^{2}\right)}{2 E_{1} E_{2}} \delta\left(E-E_{1}-E_{2}\right) \\
& \times z_{q}^{2} \mathrm{e}^{-\left(E_{1}+E_{2}\right) / T}\left\{p_{1}\right\}\left[-\frac{|\vec{p} 1|^{2}}{2|\vec{p}|^{2}}+\frac{3\left(\vec{p} \cdot \vec{p}_{1}\right)}{2|\vec{p}|^{4}}\right] \\
= & \frac{E}{4} \frac{\mathrm{d} R_{0}{ }^{l^{+} l^{-}}}{\mathrm{d}^{4} p}
\end{aligned}
$$

and

$$
\begin{aligned}
\mathscr{N}= & \int \frac{\mathrm{d}^{3} \vec{p}_{1}}{(2 \pi)^{6}} \frac{M^{2} g^{2} \sigma\left(M^{2}\right)}{2 E_{1} E_{2}} \delta\left(E-E_{1}-E_{2}\right) \\
& \times z_{q}^{2} \mathrm{e}^{-\left(E_{1}+E_{2}\right) / T}\left\{T^{2} \partial_{T} \ln \left(z_{q}\right)\right\}\left[-\frac{\left|\overrightarrow{p_{1}}\right|^{2}}{2|\vec{p}|^{2}}+\frac{3\left(\vec{p} \cdot \vec{p}_{1}\right)}{2|\vec{p}|^{4}}\right] \\
= & \frac{1}{3} T^{2} \partial_{T} \ln \left(z_{q}\right){\frac{\mathrm{d} R_{0}}{\mathrm{~d}^{4} p}}^{l^{+} l^{-}} .
\end{aligned}
$$

The non-equilibrium contribution to the dilepton rates can be written using Eqs. (33) and (32) as

$$
\begin{aligned}
{\frac{\mathrm{d} R_{1}}{\mathrm{~d}^{4} p}}^{l^{+} l^{-}}= & -\frac{8}{9} \frac{1}{\hat{q}_{q} T}\left[\frac{E}{4}+\frac{1}{3} T^{2} \partial_{T} \ln \left(z_{q}\right)\right] \\
& \times \frac{\mathrm{d} R_{0}{ }^{l^{+} l^{-}}}{\mathrm{d}^{4} p} \quad p_{i} p_{j} \Delta u_{i j} .
\end{aligned}
$$

Finally, we write the expression for the total dilepton production, using Eq. (25), as

$$
\begin{aligned}
\frac{\mathrm{d} R^{l^{+} l^{-}}}{\mathrm{d}^{4} p}= & {\left[1-\frac{8}{9} \frac{1}{\hat{q}_{q} T}\left(\frac{E}{4}+\frac{1}{3} T^{2} \partial_{T} \ln \left(z_{q}\right)\right) p_{i} p_{j} \Delta u_{i j}\right] } \\
& \times \frac{\mathrm{d} R_{0} l^{+} l^{-}}{\mathrm{d}^{4} p}
\end{aligned}
$$

Next, we plot the equilibrium rates $\frac{\mathrm{d}_{0} R_{0} p}{\mathrm{~d}^{4} p} l^{l^{-}}$using Eq. (27) for two different temperatures $(T=0.3$ and $T=0.2 \mathrm{GeV})$ in Fig. 1 with effective quark fugacity $z_{q}$ taken from Ref. [57] (dotted line) and with $z_{q}=1$ (solid line). The latter case corresponds to the equation of state of ultra-relativistic massless quarks and gluons (ideal). From the above figure it is clear that the effect of $z_{q}$ is to suppress the rates uniformly for all dilepton energies and suppression is more dominant at lower temperatures [55].

The authors had calculated the effect of realistic equation of state, via $z_{q}$ on dilepton production (Eq. (27)), along with the effect of shear and bulk viscosities in Ref. [55]. The dilepton production rate expression obtained in this work shows the effect of turbulence and momentum anisotropy, apart from the equation of state. Though the method of getting the rates remains the same, the non-equilibrium effect included in the distribution functions used in Ref. [55] is that of viscosities, unlike that of turbulent chromo-fields in the present work. 


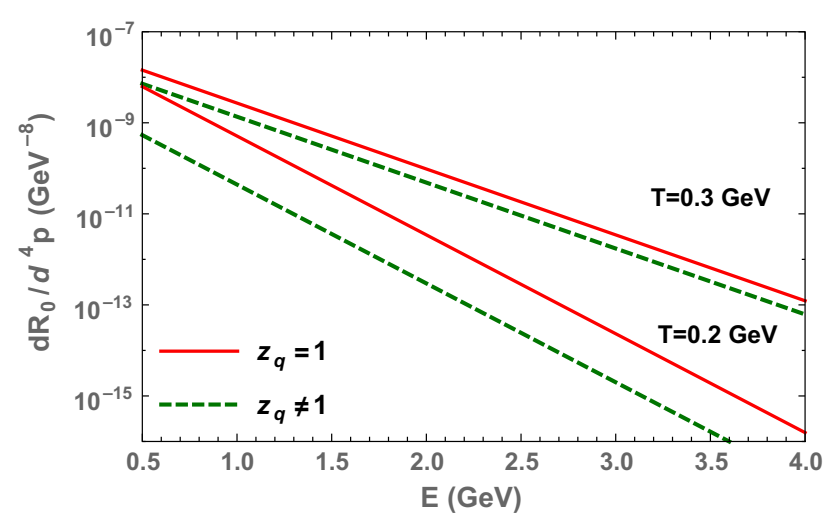

Fig. 1 The equilibrium dilepton production rates of $z_{q}^{2}$ as a function of $T / T_{c}$ is shown along with its SB limit $\left(z_{q} \rightarrow 1\right)$. The temperature dependence of the effective quark fugacity, $z_{q}$ is taken from Ref. [57]

It is noted that all above analyses were done in the rest frame of the medium and, therefore, in the general frame with four-velocity $u^{\mu}$, these results become

$$
\begin{aligned}
& {\frac{\mathrm{d} R_{0}{ }^{l^{+} l^{-}}}{\mathrm{d}^{4} p}}^{=}=\frac{z_{q}^{2}}{2} \frac{M^{2} g^{2} \sigma\left(M^{2}\right)}{(2 \pi)^{5}} \mathrm{e}^{-u \cdot p / T}, \\
& {\frac{\mathrm{d} R_{1}}{\mathrm{~d}^{4} p}}^{l^{+} l^{-}}=-\frac{8}{9} \frac{1}{T^{4}}\left(\frac{\hat{q}_{q}}{T^{3}}\right)^{-1}\left[\frac{u \cdot p}{4}+\frac{1}{3} T^{2} \partial_{T} \ln \left(z_{q}\right)\right] \\
& \times \frac{\mathrm{d} R_{0}}{\mathrm{~d}^{4} p} \quad\left[p^{\mu} p^{\nu} \Delta u_{\mu \nu}\right] \text {, }
\end{aligned}
$$

where we have kept the term $\hat{q}_{q} / T^{3}$ for phenomenological reasons stated in the previous section.

We now proceed to a study of the QGP thermal dilepton spectra from heavy-ion collisions with non-equilibrium contributions.

\section{Thermal dilepton yield from QGP during fireball evolution}

To study dilepton yield from the QGP phase in heavy-ion collisions, we need to model expansion of the thermalized fireball. This can be done using relativistic hydrodynamics. In this qualitative analysis, we use the longitudinal boost invariant flow model of Bjorken [88] to describe the expanding system. In the Bjorken flow, with the parametrization $t=\tau$ $\cosh \eta_{s}$ and $z=\tau \sinh \eta_{s}$, with the proper time $\tau=\sqrt{t^{2}-z^{2}}$ and space-time rapidity $\eta_{s}=\frac{1}{2} \ln \left[\frac{t+z}{t-z}\right]$, the four-velocity of the medium is written as $u^{\mu}=\left(\cosh \eta_{\mathrm{s}}, 0,0, \sinh \eta_{\mathrm{s}}\right)$. Neglecting the effects of the viscosity, now we can write the energy dissipation equation for the system as [88]

$\frac{\mathrm{d} \varepsilon}{\mathrm{d} \tau}+\frac{\varepsilon+P}{\tau}=0$
Here $\varepsilon$ is the energy density and $P$ is the pressure of the system. The above equation needs to be closed by the equation of state (EoS). We use a recent lattice QCD EoS [58] for this purpose. We take the transition temperature $T_{C}$, denoting the end of the QGP phase, as $180 \mathrm{MeV}$ in this analysis. By providing the initial conditions i.e. $\tau_{0}=0.5 \mathrm{fm} / \mathrm{c}$ and $T\left(\tau_{0}\right)=300$ $\mathrm{MeV}$ relevant for RHIC energies, we now solve the energy dissipation equation numerically to obtain the temperature profile $T(\tau)$.

Equipped with the temperature dependent thermal dilepton production rates, the dilepton yield from the QGP can be obtained by integrating these rates over the space-time history of the fireball evolution,

$$
{\frac{\mathrm{d} N}{\mathrm{~d}^{4} p}}^{l^{+} l^{-}}=\int \mathrm{d}^{4} x{\frac{\mathrm{d} R}{\mathrm{~d}^{4} p}}^{l^{+} l^{-}} .
$$

The four-volume element within the Bjorken model is given by $\mathrm{d}^{4} x=\pi R_{A}^{2} \mathrm{~d} \eta_{s} \tau \mathrm{d} \tau$. Here $R_{A}=1.2 A^{1 / 3}$ is the radius of the nucleus used for the collision and for $A u, A=197$. We parametrize the four-momentum of the dilepton as $p^{\alpha}=$ $\left(m_{T} \cosh y, p_{T} \cos \phi_{p}, p_{T} \sin \phi_{p}, m_{T} \sinh y\right)$ with $m_{T}^{2}=p_{T}^{2}+$ $M^{2}$. The factors appearing in the rate expressions i.e. Eq. (38) to be used in the above integral are given as $u . p=$ $m_{T} \cosh \left(y-\eta_{s}\right)$ and

$p^{\alpha} p^{\beta} \Delta u_{\mu \nu}=-\frac{M^{2}}{3 \tau}-\frac{m_{T}^{2}}{\tau} \sinh ^{2}\left(y-\eta_{s}\right)$.

The desired dilepton yields in terms of invariant mass $M$, transverse momentum $p_{T}$ and momentum rapidity $y$ are now given by

$$
\begin{aligned}
\frac{\mathrm{d} N^{l^{+} l^{-}}}{\mathrm{d} M^{2} \mathrm{~d}^{2} p_{T} \mathrm{~d} y} & =\pi R_{A}^{2} \int_{\tau_{0}}^{\tau_{f}} \mathrm{~d} \tau \tau \int_{-\infty}^{\infty} \mathrm{d} \eta_{s} \frac{1}{2} \frac{\mathrm{d} R}{\mathrm{~d}^{4} p} \\
& =\frac{\mathrm{d} N_{0}^{l^{+} l^{-}}}{\mathrm{d} M^{2} \mathrm{~d}^{2} p_{T} \mathrm{~d} y}+\frac{\mathrm{d} N_{1}^{l^{+} l^{-}}}{\mathrm{d} M^{2} \mathrm{~d}^{2} p_{T} \mathrm{~d} y}
\end{aligned}
$$

After performing the $\eta_{s}$ integration, the equilibrium and non-equilibrium contributions to the total dilepton yield are obtained:

$$
\begin{aligned}
\frac{\mathrm{d} N_{0}^{l^{+} l^{-}}}{\mathrm{d} M^{2} \mathrm{~d}^{2} p_{T} \mathrm{~d} y}= & \mathscr{R} \int_{\tau_{0}}^{\tau_{f}} \tau \mathrm{d} \tau z_{q}^{2} 2 K_{0}\left(z_{m}\right), \\
\frac{\mathrm{d} N_{1}^{l^{+} l^{-}}}{\mathrm{d} M^{2} \mathrm{~d}^{2} p_{T} \mathrm{~d} y}= & -\frac{8}{9} \mathscr{R} \int_{\tau_{0}}^{\tau_{f}} \mathrm{~d} \tau \frac{z_{q}^{2}}{T^{4}}\left(\frac{\hat{q}_{q}}{T^{3}}\right)^{-1} \mathscr{T}(T, p), \\
\text { with, } \mathscr{T}(T, p)= & K_{0}\left(z_{m}\right)\left\{\frac{1}{3} T^{2} \partial_{T} \ln \left(z_{q}\right)\left(m_{T}^{2}-\frac{2}{3} M^{2}\right)\right\} \\
& +K_{1}\left(z_{m}\right)\left\{\frac{m_{T}^{3}}{8}-\frac{1}{6} M^{2} m_{T}\right\} \\
& -K_{2}\left(z_{m}\right)\left\{\frac{1}{3} T^{2} \partial_{T} \ln \left(z_{q}\right) m_{T}^{2}\right\}
\end{aligned}
$$




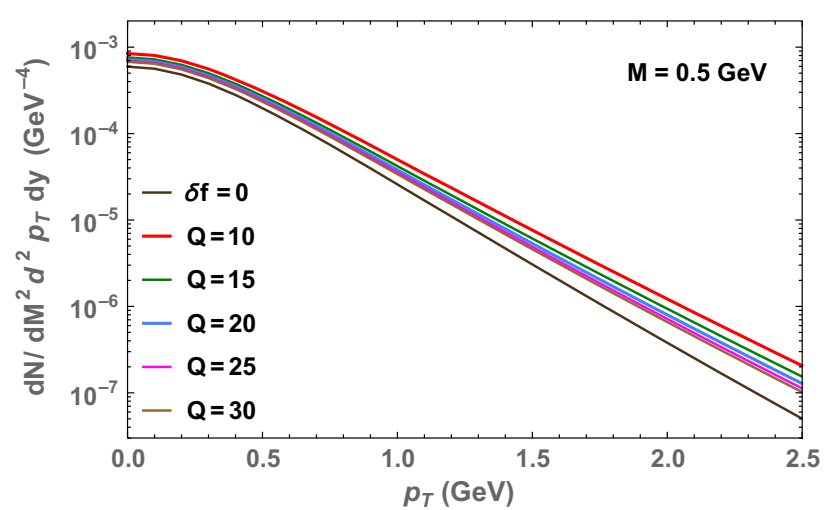

Fig. 2 Dilepton yields with non-equilibrium effects for different $\hat{q}_{q} / T^{3} \equiv Q$ values for $M=0.5 \mathrm{GeV}$. The equilibrium yield contribution is also plotted $(\delta f=0)$

$$
-K_{3}\left(z_{m}\right)\left\{\frac{m_{T}^{3}}{8}\right\},
$$

and, $\mathscr{R}=\left[\frac{\pi R_{A}^{2}}{2^{2}} \frac{1}{(2 \pi)^{5}} \frac{80 \pi \alpha^{2}}{9}\right]$.

Here the $K_{n}$ are the modified Bessel functions of the second kind and $z_{m} \equiv m_{T} / T$. Now we numerically integrate the above integrals with the temperature profile obtained from a hydrodynamical analysis to get the dilepton yields. All the results are presented for the midrapidity region of the dilepton i.e. $y=0$.

The thermal dilepton yields as a function of transverse momentum of the dileptons for the invariant mass $M=0.5$ $\mathrm{GeV}$ are shown in Fig. 2. The non-equilibrium effects are included with various values of the jet-quenching parameter, $\hat{q}_{q} / T^{3}$. The equilibrium contribution alone is also plotted $(\delta f=0)$ for comparison. It can be seen that the effect of nonequilibrium terms is to enhance the dilepton spectra throughout the $p_{T}$ regime. Also, as we increase the value of $\hat{q}_{q} / T^{3}$, the yield decreases and approaches the equilibrium value. From Eqs. (38) and (41), it is clear that the non-equilibrium contribution to dilepton rates is additive, hence we see an increase in the yields with the inclusion of non-equilibrium terms.

Notably, with $\hat{q}_{q} / T^{3}=15$, we observe $\sim 38 \%$ enhancement at $p_{T}=0.5 \mathrm{GeV}$ and $\sim 147 \%$ at $p_{T}=2 \mathrm{GeV}$. Since the $\hat{q}_{q} / T^{3}$ terms appear in the denominator of the yield expression, as seen in Eq. (43), increasing its value will result in the decrease of non-equilibrium contribution.

For e.g. $\hat{q}_{q} / T^{3}=25$, the enhancement is only about $\sim 23 \%$ and $\sim 88 \%$ for the transverse momenta 0.5 and $2 \mathrm{GeV}$, respectively.

It is crucial to note that enhancement of the spectra is more significant at high $p_{T}$, indicating the strong non-equilibrium effects at that regime. In heavy-ion collisions, high $p_{T}$ particles are produced during the initial stages of the evolution.

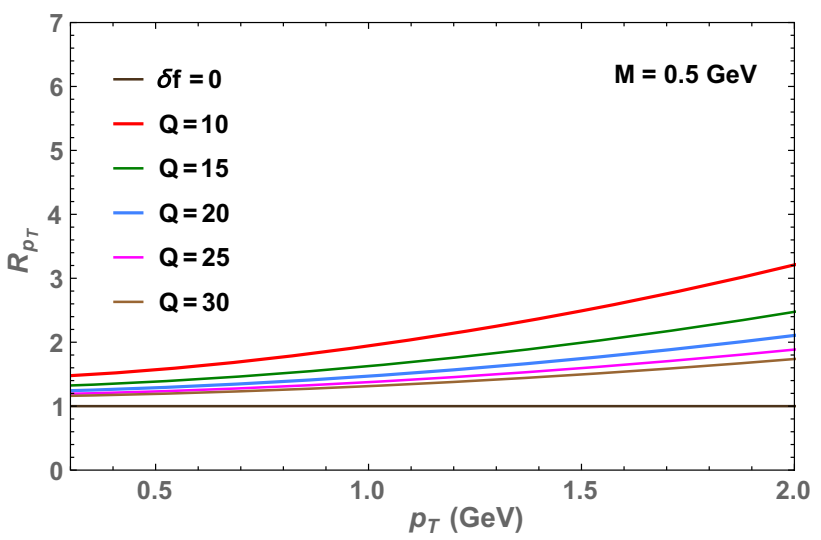

Fig. 3 Strength of non-equilibrium corrections to equilibrium yield represented through ratio $R_{p_{T}}$ (Eq. 44) for different jet-quenching parameters

Since we expect the anisotropic effects also to be dominant at the initial stages of the evolution, its effect will be more effective at high $p_{T}$. The fact that enhancement is seen in low $p_{T}$ particles indicate that the non-equilibrium effects remain significant throughout the evolution of the system. We note that just like the overall effect of viscosities as seen in Ref. [55], the present non-equilibrium effect also enhances the thermal dilepton spectra.

Next, we study the strength of these non-equilibrium corrections to the equilibrium distribution functions by looking into their contributions to dilepton spectra. We begin our analysis by constructing the following ratio:

$R_{p_{T}}=\frac{\mathrm{d} N^{l^{+} l^{-}}}{\mathrm{d} M^{2} \mathrm{~d}^{2} p_{T} \mathrm{~d} y} / \frac{\mathrm{d} N_{0}^{l^{+} l^{-}}}{\mathrm{d} M^{2} \mathrm{~d}^{2} p_{T} \mathrm{~d} y}$,

where the numerator includes non-equilibrium contributions. We plot this ratio as a function of transverse momenta of the dileptons for different $\hat{q}_{q} / T^{3}$ values in Fig. 3 .

Note that, for smaller value of the transverse momentum, the non-equilibrium contribution is changing the equilibrium part by $\sim 50 \%$ for $\hat{q}_{q} / T^{3}>10$. These non-equilibrium contributions tend to increase strongly as we move towards higher $p_{T}$. The corrections start decreasing as we increase the $\hat{q}_{q} / T^{3}$ parameter as expected, since high values of $\hat{q}_{q} / T^{3}$ dilute the non-equilibrium corrections. For $\hat{q}_{q} / T^{3}=20(30)$, the contribution begins with $\sim 28(19) \%$ at $p_{T}=0.5 \mathrm{GeV}$ and reaches $\sim 110(74) \%$ by $p_{T}=2 \mathrm{GeV}$. Overall, we observe strong corrections to the yield by non-equilibrium effects.

It is to be emphasized that, for low values of $\hat{q}_{q} / T^{3}$, significant corrections to the spectra are seen. These strong corrections due to perturbative non-equilibrium effects are indicative of the fact that such $\hat{q}_{q} / T^{3}$ values are preferably ruled out within the present model. However, to substantiate the claim thoroughly, one may need to perform a quantitative 


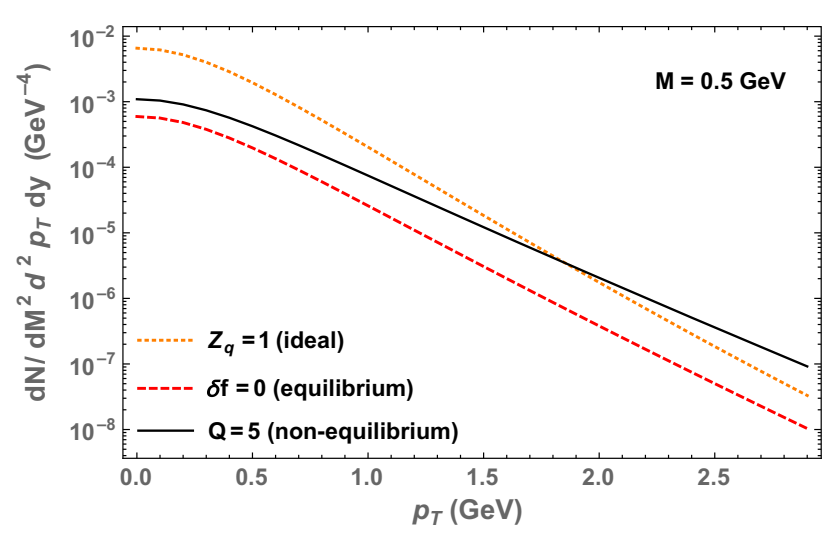

Fig. 4 Strength of non-equilibrium terms via the ratio $R_{p_{T}}$

analysis including three-dimensional hydrodynamical flow, which is beyond the scope of this paper.

Moreover, we recall that, in the present work, the corrections to the spectra are calculated within one-dimensional Bjorken flow, which is well known to over-estimate the particle spectra. So the corrections shown in this qualitative study act only as upper bounds. The precise nature of the corrections to the particle yields depends very much upon the geometry under consideration (here: the simplified Bjorken case), because of the involved space-time integration (which we perform numerically). How a different, more realistic three-dimensional geometry involving transverse flow will change the overall corrections cannot be guessed. Although it is expected that the effect of transverse flow is to decrease the particle yield, since the evolution time and, therefore, the limit of the proper time integration will be less in that case. However, the space part integration contribution is non-trivial and may change significantly under a different geometry.

Finally, let us analyze how the non-equilibrium corrections and equilibrium values are modifying the ideal case i.e. $z_{q}=1$. The yield corresponding to the ideal case can be obtained by considering Eq. (27) with $z_{q}=1$. The effect of the $z_{q}^{2}$ term on the ideal spectra was analyzed by the authors in detail elsewhere [55] and will not be repeated here. We recall that its effect was found to be suppressive and this can also be inferred from Fig. 1 of this manuscript. In Fig. 4, we plot the equilibrium and non-equilibrium dilepton yield for $M=0.5$ $\mathrm{GeV}$ with that for the ideal case. For the non-equilibrium part, we have taken the jet-quenching parameter value as $\hat{q}_{q} / T^{3}=5$ (This is typically the value that we obtain by averaging the predictions of all these approaches [93,95-99].) For low transverse momenta, non-equilibrium enhancement of equilibrium spectra is marginal compared to the ideal values. We observe that the non-equilibrium effects can overtake the $z_{q}$ suppressions of the ideal spectra $\left(z_{q}=1\right)$ at high $p_{T} \mathrm{~s}$. At $p_{T} \sim 2 \mathrm{GeV}$, we have near-cancellation of these effects. However, thereafter non-equilibrium contribution will domi- nate the other two. However, by increasing the jet-quenching parameter, the strength of the $z_{q}$ suppressions can be made to dominate so that the total spectra will become less than the ideal case.

It is to be emphasized that in the present, qualitative study, we have used a one-dimensional Bjorken flow to model the system. It is well known that the Bjorken results tend to over-estimate the particle production because the system takes more time to cool down compared to a realistic threedimensional expansion with the transverse flow [53]. However, such a quantitative study is not within the scope of the present analysis and will be taken up for investigation in the near future. It is encouraging that we are seeing very significant effects with one-dimensional flow, which, we believe, may guide us to finding observable signatures on realistic three-dimensional calculations.

\section{Conclusions and outlook}

In conclusion, thermal particle production is investigated in the presence of momentum anisotropy during the hydrodynamical expansion of the QGP in heavy-ion collisions. The effects of anisotropy are encoded in the non-equilibrium part of the quark/antiquarks and gluon momentum distribution functions. We particularly study the dilepton production rate and compare our results against the isotropic/equilibrium case. The modifications induced by the anisotropy are found to be significant as far as the rate and dilepton yields are concerned. The strength of the anisotropy, which in our case is inversely proportional to the jet-quenching parameter apart from other momentum dependent factors, has appreciable impact on the rate and yield. The whole analysis is based on an effective transport equation which is obtained by an ensemble averaging of the turbulent gluonic fields created due to the momentum anisotropy by inducing instability in the $S U$ (3) Yang-Mills equations. We can perhaps treat the thermal particle production in the presence of the momentum anisotropy as an indicator of the impact of turbulent color fields and anomalous transport processes during the expansion of QGP.

It would be interesting to include collisional processes and study the interplay of them with anomalous ones by setting up and solving an appropriate transport equation for the nonequilibrium distributions. We intend to employ them to study thermal particle production in heavy-ion collisions.

Acknowledgements This work has been conducted under the INSPIRE Faculty grant of Dr. Vinod Chandra (Grant No. IFA-13/ PH-55, Department of Science and Technology, Govt. of India) at Indian Institute of Technology Gandhinagar, India. We would like to record our sincere gratitude to the people of India for their generous support for the research in basic sciences in the country. 
Open Access This article is distributed under the terms of the Creative Commons Attribution 4.0 International License (http://creativecomm ons.org/licenses/by/4.0/), which permits unrestricted use, distribution, and reproduction in any medium, provided you give appropriate credit to the original author(s) and the source, provide a link to the Creative Commons license, and indicate if changes were made.

Funded by SCOAP ${ }^{3}$.

\section{References}

1. J. Adams et al., STAR collaboration, Nucl. Phys. A 757, 102 (2005)

2. K. Adcox et al., PHENIX Collaboration, Nucl. Phys. A 757, 184 (2005)

3. B. Back et al., PHOBOS Collaboration, Nucl. Phys. A 757, 28 (2005)

4. A. Arsence et al., BRAHMS Collaboration, Nucl. Phys. A 757, 1 (2005)

5. K. Aamodt et al., (The Alice Collaboration), arXiv:1011.3914 [nucl-ex]

6. K. Aamodt et al., The Alice Collaboration, Phys. Rev. Lett. 105, $252301(2010)$

7. K. Aamodt et al., The Alice Collaboration, Phys. Rev. Lett. 106, 032301 (2011)

8. L. Del Zanna et al., Eur. Phys. J. C 73, 2524 (2013)

9. V. Rolando et al., Nucl. Phys. A 931, 970-974 (2014)

10. F. Becattini et al., Eur. Phys. J. C 75, 406 (2015)

11. B. Schenke, S. Jeon, C. Gale, Phys. Rev. Lett. 106, 042301 (2011)

12. P. Bozek, Phys. Rev. C 85, 034901 (2012)

13. U. Heinz, R. Snellings, Ann. Rev. Nucl. Part. Sci. 63, 123 (2013)

14. C. Gale, S. Jeon, B. Schenke, Int. J. Mod. Phys. A 28, 1340011 (2013)

15. H. Song, U.W. Heinz, Phys. Rev. C 81, 024905 (2010)

16. H. Niemi, G.S. Denicol, P. Huovinen, E. Molnar, D.H. Rischke, Phys. Rev. Lett. 106, 212302 (2011)

17. J. Randrup, S. Mrowczynski, Phys. Rev. C 68, 034909 (2003)

18. P. Arnold, J. Lengaghan, G.D. Moore, J. High Energy Phys. 08, $002(2003)$

19. A.H. Mueller, A.I. Shoshi, S.M.H. Wong, Phys. Lett. B 632, 257 (2006)

20. S. Mrowczynski, B. Schenke, M. Strickland, Phys. Rep. (2017). doi:10.1016/j.physrep.2017.03.003

21. M. Asakawa, S.A. Bass, B. Müller, Phys. Rev. Lett. 96, 252301 (2006)

22. M. Asakawa, S.A. Bass, B. Müller, Prog. Theor. Phys. 116, 725 (2007)

23. V. Chandra, V. Ravishankar, Eur. Phys. J. C 64, 63 (2009)

24. T. Abe, K. Niu, J. Phys. Soc. Jpn. 49, 717 (1980) (ibid. 49, 725, 1980)

25. S. Mrowczynski, Phys. Lett. B214, 587 (1988) (ibid. 314, 118, 1993)

26. P. Romatschke, M. Strickland, Phys. Rev. D 68, 036004 (2003)

27. P. Arnold, J. Lengaghan, G.D. Moore, L.G. Yaffe, Phys. Rev. Lett. 94, 072302 (2005)

28. J. Berges, K. Boguslavski, S. Schlichting, Phys. Rev. D 85, 076005 (2012)

29. J. Berges, S. Scheffler, S. Schlichting, D. Sexty, Phys. Rev. D 85, 034507 (2012)

30. J. Berges, S. Scheffler, S. Schlichting, D. Sexty, Phys. Rev. D 86, 074006 (2012)

31. S. Berges, S. Schlichting, Phys. Rev. D 87, 014026 (2013)

32. J. Berges, K. Boguslavski, S. Schlichting, R. Venugopalan, J. High Energy Phys. 05, 054 (2014)
33. J. Berges, K. Boguslavski, S. Schlichting, R. Venugopalan, Phys. Rev. D 89, 074011 (2014)

34. J. Berges, K. Boguslavski, S. Schlichting, R. Venugopalan, Phys. Rev. D 89, 114007 (2014)

35. J. Berges, K. Boguslavski, S. Schlichting, R. Venugopalan, Phys. Rev. Lett. 114, 061601 (2015)

36. K. Fukushima, Phys. Rev. C 89, 024907 (2014)

37. K. Fukushima, F. Gelis, Nucl. Phys. A 874, 108 (2012)

38. A. Kurkela, G.D. Moore, arxiv:1209.4091 [hep-lat]

39. A. Kurkela, G.D. Moore, Phys. Rev. D 86, 056008 (2012)

40. Erich S. Weibel, Phys. Rev. Lett. 2, 83 (1959)

41. T.H. Dupree, Phys. Fluids 9, 1773 (1966) (ibid. 11, 2680, 1968)

42. V. Chandra, S.K. Das, Phys. Rev. D 93, 094036 (2016). arXiv: 1506.07805 [nucl-th]

43. S.K. Das, V. Chandra, J. Alam, J. Phys. G 41, 015102 (2013). arXiv: 1210.3905 [nucl-th]

44. S.K. Das, F. Scardina, S. Plumari, V. Greco, Phys. Lett. B 747, $260(2015)$

45. J. Alam, B. Sinha, S. Raha, Phys. Rep. 273, 243 (1996)

46. J. Alam, S. Sarkar, P. Roy, T. Hatsuda, B. Sinha, Ann. Phys. 286, 159 (2001). arXiv:hep-ph/9909267

47. T. Peitzmann, M.H. Thoma, Phys. Rep. 364, 175 (2002). arXiv:hep-ph/0111114

48. H. Van Hees, R. Rapp, Nucl. Phys. A 806, 339 (2008). arXiv:0711.3444 [hep-ph]

49. L.D. McLerran, T. Toimela, Phys. Rev. D 31, 545 (1985)

50. J.R. Bhatt, H. Mishra, V. Sreekanth, JHEP 1011, 106 (2010). doi:10.1007/JHEP11(2010)106. arXiv:1011.1969 [hep-ph]

51. J.R. Bhatt, V. Sreekanth, Int. J. Mod. Phys. E 19, 299 (2010). doi:10.1142/S0218301310014765. arXiv:0901.1363 [hep-ph]

52. J. Peralta-Ramos, M.S. Nakwacki, arXiv:1010.3672 [hep-ph]

53. J.R. Bhatt, H. Mishra, V. Sreekanth, Nucl. Phys. A 875, 181 (2012). arXiv:1101.5597 [hep-ph]

54. R.S. Bhalerao, A. Jaiswal, S. Pal, V. Sreekanth, Phys. Rev. C 88 044911 (2013). arXiv:1305.4146 [nucl-th]

55. V. Chandra, V. Sreekanth, Phys. Rev. D 92(9), 094027 (2015). arXiv:1511.01208 [nucl-th]

56. V. Chandra, V. Ravishankar, Eur. Phys. J. C 59, 705 (2009)

57. V. Chandra, V. Ravishankar, Phys. Rev. D 84, 074013 (2011)

58. M. Cheng et al., Phys. Rev. D 77, 014511 (2008)

59. S. Borsanyi et al., JHEP 1009, 073 (2010)

60. S. Borsanyi et al., JHEP 11, 077 (2010)

61. Y. Aoki et al., JHEP 0601, 089 (2006)

62. Y. Aoki et al., JHEP 0906, 088 (2009)

63. Vinod Chandra, Phys. Rev. D 86, 114008 (2012)

64. Vinod Chandra, Phys. Rev. D 84, 094025 (2011)

65. S. Mitra, V. Chandra, Phys. Rev. D 94, 034025. arXiv: 1606.08556 [nucl-th]

66. A. Peshier et al., Phys. Lett. B 337, 235 (1994)

67. A. Peshier et al., Phys. Rev. D 54, 2399 (1996)

68. A. Peshier, B. Kampfer, G. Soff, Phys. Rev. C 61, 045203 (2000)

69. A. Peshier, B. Kampfer, G. Soff, Phys. Rev. D 66, 094003 (2002)

70. M. DÉlia, A. Di Giacomo, E. Meggiolaro, Phys. Lett. B 408, 315 (1997)

71. M. DÉlia, A. Di Giacomo, E. Meggiolaro, Phys. Rev. D 67, 114504 (2003)

72. P. Castorina, M. Mannarelli, Phys. Rev. C 75, 054901 (2007)

73. P. Castorina, M. Mannarelli, Phys. Lett. B 664, 336 (2007)

74. A. Dumitru, R.D. Pisarski, Phys. Lett. B 525, 95 (2002)

75. K. Fukushima, Phys. Lett. B 591, 277 (2004)

76. S.K. Ghosh et al., Phys. Rev. D 73, 114007 (2006)

77. H. Abuki, K. Fukushima, Phys. Lett. B 676, 57 (2006)

78. H.M. Tsai, B. Mller, J. Phys. G 36, 075101 (2009)

79. M. Ruggieri et al., Phys. Rev. D 86, 054007 (2012)

80. P. Alba, W. Alberico, M. Bluhm, V. Greco, C. Ratti, M. Ruggieri, Nucl. Phys. A 934, 41-51 (2015) 
81. S. Jeon, Phys. Rev. D 52, 3591 (1995)

82. S. Jeon, L.G. Yaffe, Phys. Rev. D 53, 5799 (1996)

83. M. Bluhm, B. Kampfer, K. Redlich, Nucl. Phys. A 830, 737c$740 \mathrm{c}(2009)$

84. K. Dusling, T. Schäfer, Phys. Rev. C 77, 034905 (2008)

85. K. Dusling, D. Teaney, Phys. Rev. C 85, 044909 (2012)

86. M. Bluhm, B. Kampfer, R. Schulze, D. Seipt, U. Heinz, Phys. Rev. C 76, 034901 (2007)

87. P. Chakraborty, J.I. Kapusta, Phys. Rev. C 83, 014906 (2011)

88. J.D. Bjorken, Phys. Rev. D 27, 140 (1983)

89. U.W. Heinz, Phys. Rev. Lett. 51, 351 (1983)

90. U.W. Heinz, Ann. Phys. 161, 48 (1985)

91. U.W. Heinz, Ann. Phys. 168, 148 (1986)

92. A. Majumdar, B. Muller, X.-N. Wang, Phys. Rev. Lett. 99, 192301 (2007)

93. K.M. Burke et al., arXiv:1312.5003v2 [nucl-th]

94. M. Asakawa, S.A. Bass, B. Müller, Nucl. Phys. A 854, 76-80 (2011)

95. A. Buzzatti, M. Gyulassy, Phys. Rev. Lett. 108, 022301 (2012)

96. X.-F. Chen, T. Hirano, E. Wang, X.-N. Wang, H. Zhang, Phys. Rev. C 84, 034902 (2011). arXiv:1102.5614 [nucl-th]

97. A. Majumder, C. Shen, Phys. Rev. Lett. 109, 202301 (2012). arXiv:1103.0809 [hep-ph]

98. B. Schenke, C. Gale, S. Jeon, Phys. Rev. C 80, 054913 (2009). arXiv:0909.2037 [hep-ph]

99. G.Y. Qin, J. Ruppert, C. Gale, S. Jeon, G.D. Moore, M.G. Mustafa, Phys. Rev. Lett. 100, 072301 (2008). arXiv:0710.0605 [hep-ph]
100. S. Mrowczynski, Phys. Lett. B 314, 118-121 (1993)

101. S. Mrowczynski, Phys. Rev. C 49, 2191-2197 (1994)

102. S. Mrowczynski, Phys. Lett. B 393, 26-30 (1997)

103. P. Arnold, J. Lenghan, G.D. Moore, L.G. Yaffe, Phys. Rev. Lett. 94, 072302 (2005)

104. A. Rebhan, P. Romatschke, M. Strickland, Phys. Rev. Lett. 94, 102303 (2005)

105. P. Romatschke, R. venugopalan, Phys. Rev. Lett. 96, 062302 (2006)

106. P. Romatschke, M. Strickland, Phys. Rev. D 70, 116006 (2004)

107. M.E. Carrington, K. Deja, S. Mrowczynski, Phys. Rev. C 90(3), 034913 (2014)

108. M. Yousuf Jamal, S. Mitra, V. Chandra, Phys. Rev. D 95, 094022 (2017)

109. P. Romatschke, M. Strickland, Phys. Rev. D 71(12), 125008 (2005)

110. P. Romatschke, Phys. Rev. C 75(1), 014901 (2007)

111. P. Roy, A.K. Dutt-Mazumder, Phys. Rev. C 83(4), 044904 (2011)

112. J. Ruppert, B. Muller, Phys. Lett. B 618, 123-130 (2005)

113. P. Chakraborty, M.G. Mustafa, M.H. Thoma, Phys. Rev. D 74(9), 094002 (2006)

114. M. Mandal, P. Roy, Adv. High Energy Phys. 2013, 371908 (2013)

115. R. Vogt, Ultrarelativistic Heavy-Ion Collisions (Elsevier, New York, 2007) 